\title{
Pragmatic Failures and Countermeasures Analysis in Intercultural Communication Context
}

\author{
Zhe $\mathrm{Li}^{1, \mathrm{a}}$, Yanming $\mathrm{Li}^{2, \mathrm{~b}}$, Deyin Yao ${ }^{3, \mathrm{c}}$ \\ ${ }^{1}$ College of International Exchange, Bohai University, Jinzhou, 121013, China \\ ${ }^{2}$ School of Applied Technology, University of Science and Technology Liaoning, Anshan, 111400, \\ China \\ ${ }^{3}$ College of Engineering, Bohai University, Jinzhou, 121013, China \\ a503412508 @qq.com, basliyanming@163.com, cdeyinyao2013@gmail.com
}

Keywords: intercultural communication; context; pragmatic failure; countermeasures; manifestation

\begin{abstract}
Pragmatic failure is people of different cultural background communicate frequent phenomenon. If to meet and understand each other's culture, communication is not smooth is likely to happen, which can lead to pragmatic failure, affect the communication smoothly. Communication is always in a certain language environment, the research of the context of intercultural communication pragmatic failure has a certain practical significance. First, the composed of a variety of contexts context hierarchy system; Then, the study of pragmatic failure forms, including language, social pragmatic failures pragmatic mistakes and pragmatic behavior; Finally, the paper puts forward the context of intercultural communication pragmatic failures to prevent countermeasures, including develop two-way communication, appropriate language use regulation to culture teaching strategies and teaching strategies.
\end{abstract}

\section{Introduction}

Context is short for language environment, is refers to the environment at the time of the communication or scenarios, including natural context, local context and human context. Natural context refers to the communicative language speakers living environment, local context refers to the communicator in working or living part time in the target language environment, human context refers to the communicator using the target language to build the scene in your mind. Communication needs to be done in a certain scene and the ravers' social role and social communication and communication purpose directly affect the communication behaviors. Communication of different cultures in the context of intercultural communication is much difficult, often cause pragmatic failure. Pragmatic failure is in the process of communication, while the speaker USES the words and grammar are correct language, but if does not conform to the context at that time, did not consider the communication on both sides of the identity and status, and against social statute and the values of the target language, communication failures of this nature.

The causes of pragmatic failure is very much, simple summary is the following: the communicator lack sufficient knowledge of each other's culture, don't know oneself what is appropriate behavior in this scene, so as to produce psychological anxiety; At the same time, the other person's behavior patterns and their cultural norms of this kind of situation is different, resulting in an aversion; Lack of knowledge communication and the influence of the negative emotions from behavior has displayed the appropriate communication skills, no positive communication motivation and attitude. In this paper, based on the cross-cultural communication theory and context theory study of pragmatic failures, put forward the precaution countermeasure against the context of intercultural communication, in order to improve the cross-cultural communicative competence to provide theoretical guidance and practical method. 


\section{Structure and Function of Context}

Context in pragmatics and discourse entities and pragmatic subject is in one of the three major elements, is a relatively independent existence, a certain system structure in itself. Many factors have appropriate structural hierarchy and each upper context contains more than one lower level context, elaborating for the bottom of the context is the upper context. Different contextual factors in the role of specific pragmatic activities are not entirely equivalent, the composition of contextual factors with its function and specific pragmatic actions together. In pragmatic communication context factors constitutes the wide coverage, the structure of system structure of the orderly, clear and distinct, as shown in Fig. 1.

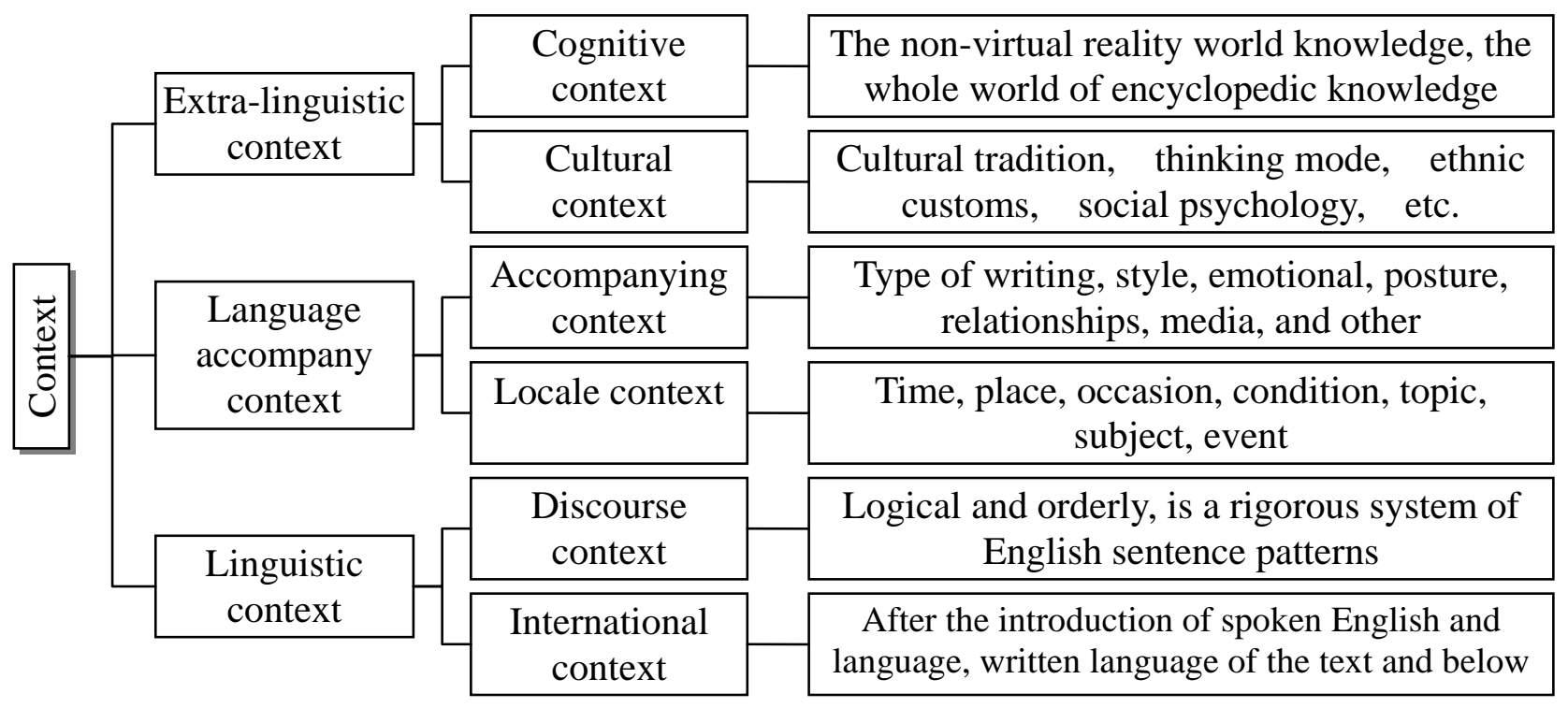

Fig. 1. Structure system of context

The context structure should have the thought of dialectical thinking [1]: a contextual factors must with pragmatic behavior, activities and processes such as closely related to and important influence on pragmatic itself; Contextual factors in the process of concrete pragmatic can grasp and analysis; To pay attention to the structural factors of context, the permeability of external and internal structure of order structure is the organic unity; In the real communication, context factors in dynamically generated and constantly changing.

Context in the process of communication function mainly embodied in two aspects: one is the restriction function, information transmission in the process of communication is always in a certain context, a certain context as rely on and context of language use is produce restrictive function. Restriction function is for language hair, hair person must have the corresponding ability to adapt, according to the context of words to make the corresponding adjustment; 2 it is to explain the function, may only be simple literal meaning, language expression may be conferred by the context of deep meaning, it is also possible that the implication. Deep meaning and implication is closely related to the social scene, need of implied meaning explanation context. Explain function is for the words, can according to the context to get the corresponding explanation.

\section{Manifestations of Pragmatic Failure}

The British linguist Jcnny Thomas cross-cultural pragmatic failures can be divided into language and social pragmatic failures of two kinds of pragmatic failures. Combining previous research results, this article will form of pragmatic failures in cross-cultural communication as the "language, social pragmatic failures please use mistakes and pragmatic behavior" three types, structure as shown in Fig. 2. 
(1) Language pragmatic failure. Communication will be in the native language pragmatic meaning of a word or structure, to paraphrase in the target language caused by the error, which conveys the intended to convey the implication. No absolute equivalence between any two languages vocabulary, some language of expression will be because of geographical location, social customs, way of life such as different and adopt different expression [2].Specific performance is the speaker thinks the hearer can fully understand your words, the hearer make wrong pragmatic reasoning.

(2) Social pragmatic failure. Come from different cultural differences of way of thinking and customs, do not understand each other's social and cultural background, language use and understanding error, communication cannot achieve the desired effect [3].Do not understand cultural background, to their own special culture and consciousness, through the form of language upon each other, and without regard to the language conform to each other's habits and social norms. Social pragmatic failure is originated in the cultural conflict, related to people's values.

(3) The Pragmatic behaviors failure. Pragmatic behavior that nonverbal behavior of communication, through the use of words does not belong to the category of methods to transfer information or pleasure. Pragmatic behavior form covers all the language category, such as expression, action, posture, instrument, such as sounds and smells, keep the distance of the communication process, silence in the process of language communication time and communication, etc. Because of cultural differences silent non-verbal behavior and present different meanings, different communication effect $[4,5]$.

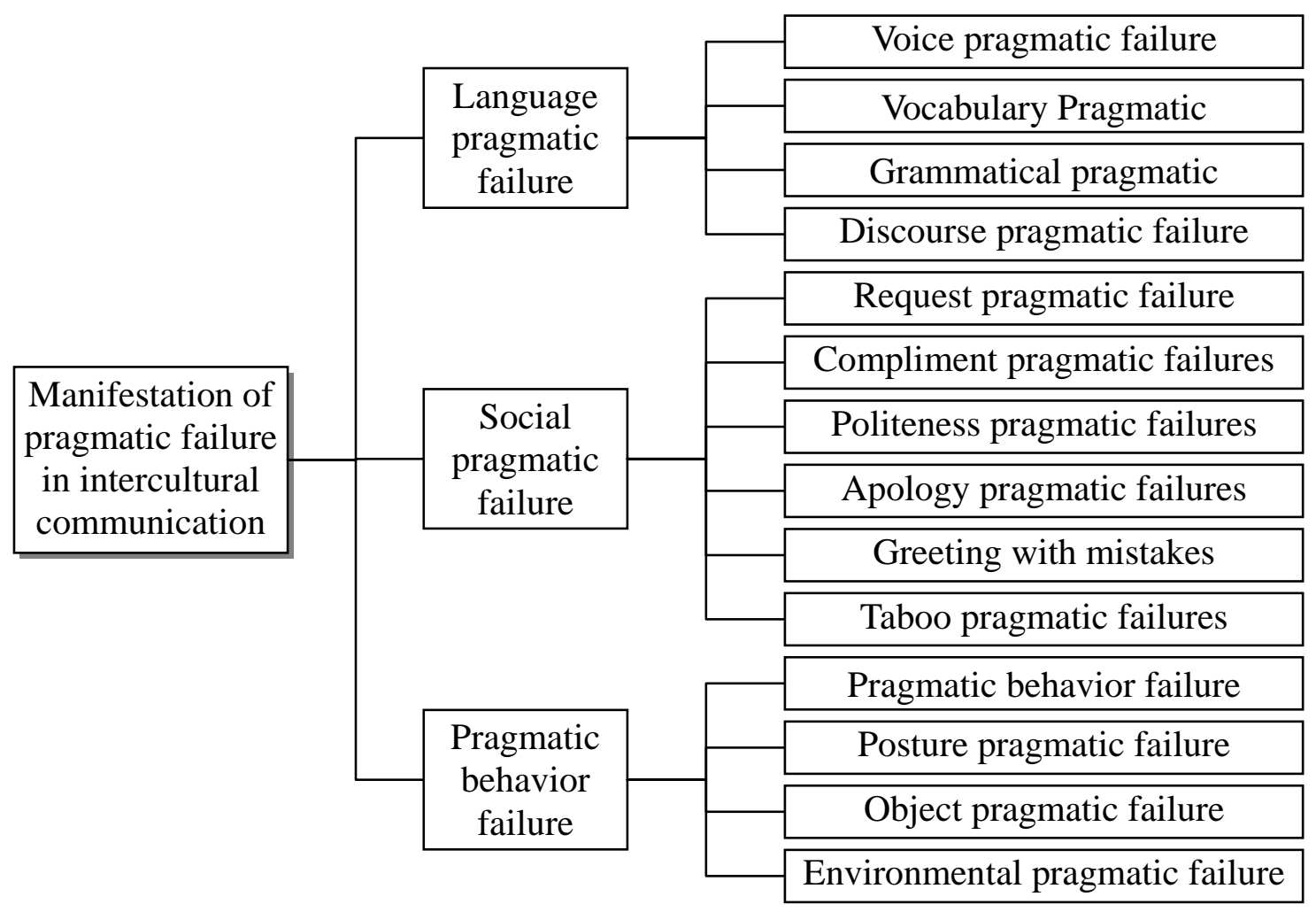

Fig. 2. Manifestations structure of pragmatic failure

\section{Countermeasures Analysis in Intercultural Communication Context}

Communication is between people communicate thoughts and feelings of social activities. Sender and receiver are dependent on the specific context in communication and restricted by context, neglect the contextual adaptation, can lead to communication failure. On the structure and function of the context and pragmatic failure forms, the context of intercultural communication pragmatic failures to prevent countermeasures are as follows: 
(1) Develop two-way communication ability. Two-way communication refers to the position between sender and recipient constantly exchange, and the attitude of the sender to negotiation or discussion in the face of the receiver, information issue need to listen to feedback in time, after both sides can be repeated if necessary, until to clear and satisfaction. Two-way communication process as shown in Fig. 3 [6]. Is common in the process of cross-cultural communication, communication, but communication is not the same as understanding. Communication both sides involved in coding and decoding, through the feedback information, can further clarify intent. Advantage is to communicate information accuracy is high, the recipient feedback opportunity to, can produce equal feeling and engagement. Two-way communication in the context of real, can be a verbal expression, also can is non-verbal expression. Oral communication should pay attention to clear and slow, repetitive, simple, and the main dynamic; Nonverbal expression should pay attention to visual repetition, gestures, such as demonstration, pause and summarizes the strategies. At the same time, pay attention to distinguish, do not be eager to break the silence, find out the differences, check for understanding.

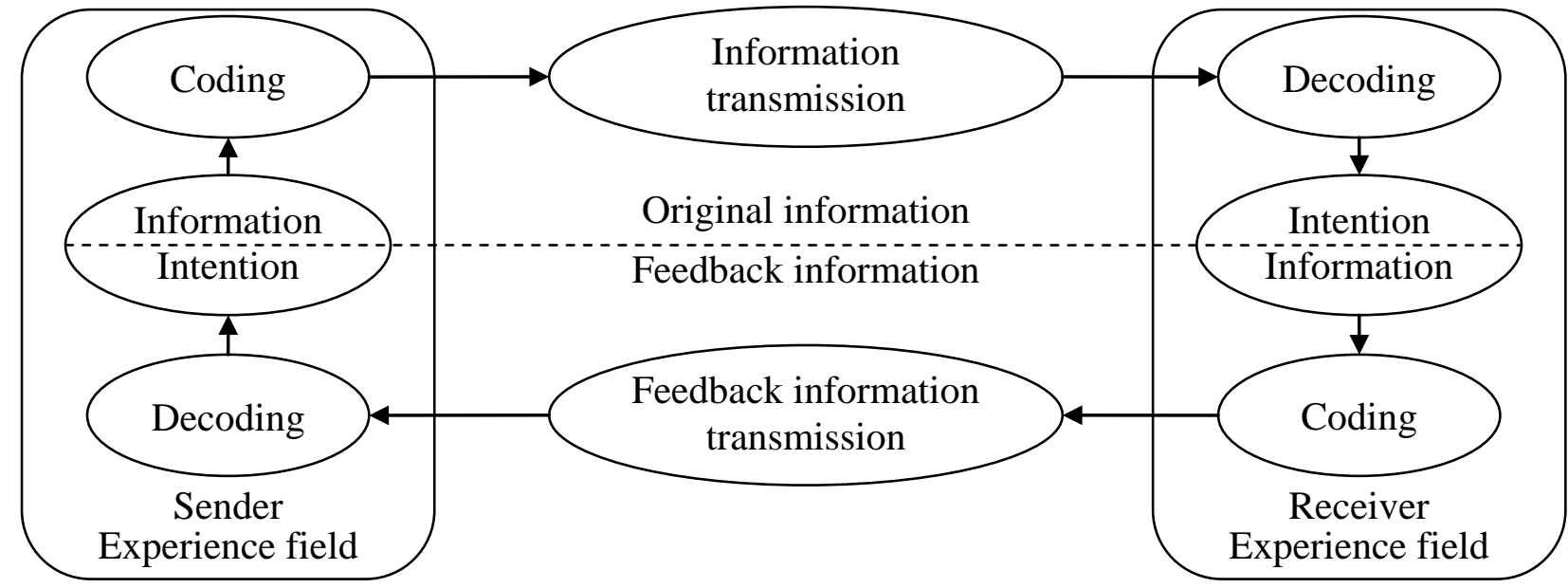

Fig. 3. Two-way communication process

(2) The appropriate language use strategies [7]. In the process of communication, people are not adapt to the context blindly, sometimes need to break the bondage of inherent context, creating context, create favorable communication context. Pragmatic subject can adopt appropriate pragmatic strategies for realizing the function of contextual phonetic, semantic and syntax elements to regulate or control, to achieve the purpose of smooth communication. Voice control strategy, the voice of pragmatic subject using context in verbal communication performance characteristics, consciously on phonetic context or construction, or adjusted, or to the means of control; Semantic control strategy, polysemy is language significance the inevitable result of the contradiction and semantic evolution, in multiple meanings once in specific context, will produce ambiguity is restricted by context, to adjust and control can make use of semantic ambiguity, also can use semantic distortion control measures; Topic control strategy, pragmatic subject according to the communicative purpose, depending on the topic to build different context, consciously choose topic or change the subject, to construct suitable strategy context to achieve communication is smooth.

(3) Take the cultural teaching strategies in teaching. Along with the education to deepen the understanding of language rich cultural connotation, intercultural education has become the necessary link of the foreign language teaching. Foreign language teaching is not only the language knowledge, but also culture teaching strategies should be taken, through cultural knowledge, enriching the connotation of the foreign language learning, stimulate interest in learning, active classroom atmosphere, to cultivate and enhance the cultural communication and language skills [8].Culture teaching strategies from the following aspects: the cultural differences between mother tongue and target language are compared; Make full use of images, animation, audio and video and other multimedia teaching methods, create transfer students feel the atmosphere of foreign culture; Guide the student to read the original foreign literature, the perspective of the target language 
countries cultural nature, cultural characteristics and the author's psychological state; Make full use of the classic textbook insight into the cultural connotation of the target language countries; Teach cultural knowledge for students to create a certain situation; Hiring foreign teachers teach for students cross-cultural communication course; All kinds of extracurricular activities for the students to make students experience a foreigner behavior and the way to express one's thoughts and feelings.

\section{Conclusion}

With the rapid development of economic globalization, frequent exchanges between China and the world, if you don't understand the cultural background of the communication on both sides, the difference of way of thinking, value orientation and so on, can cause many pragmatic failures, communication both sides appear embarrassed, misunderstanding, etc. and will inevitably affect the communication. Pragmatic failure as one of the important factors that affect the cross-cultural communication smoothly, has attracted extensive attention of linguists. Cross-cultural communication can help the communicator through communication with different cultural backgrounds can better understand and know each other, to exchange their consciousness to think proper and appropriate language and words may be in different cultural background will be considered inappropriate speech ACTS, and be able to timely find communication barriers and communication difficulties, know how to take remedial measures to adjust the speech act of specification, so as to avoid communication conflicts, to achieve the purpose of smooth communication. Cross-cultural communication can make people realize that there is only the correctness of the language form and language content and accuracy to attain the goal of intercultural communication is not enough. Because the same ideas and opinions can use different forms of language to express, therefore, in the process of cross-cultural communication must consider the appropriateness of language expression, what language form in the context of what use is more appropriate.

\section{Acknowledgement}

This work is supported by social science fund project of Liaoning province (L14BYY014): Research on Pragmatic Failure and Its Strategy for Intercultural Communication.

\section{References}

[1] J. H. Wang, "Constitution and Classification of Context," Applied linguistics, vol. 11, no. 3, pp. 2-9, 2002.

[2] J. Zhao, "Study of pragmatic failure in Chinese grammar learning of foreign students," Journal of Educational Institute of Jilin Province, vol. 29, no. 7, pp. 60-62, 2013.

[3] H. Liang, "An analysis of cross cultural pragmatic failure from the perspective of speech act theory," Journal of Technology, vol. 32, no. 2, pp. 5-7, 2015.

[4] S. J. Zhang, D. M. Li, "Analysis of pragmatic failure of body language based on Hofstede Geert," Hei Longjiang Education (Theory \& Practice), vol. 2, no. 10, pp. 43-44, 2014.

[5] Y. Q. Hu, "Causes and Measures of Cross - cultural Pragmatic Failure among Non-English Majors," Journal of Xinjiang Vocational University, vol. 32, no. 5, pp. 36-37, 2014.

[6] H. Liang, "Competence Cultivation of Cross\}ultural Communication in English Teaching," Foreign Language and Literature, vol. 28, no. 5, pp. 146-150, 2012.

[7] S. Dong, "On Contextual Strategy," Journal of Zhejiang Wanli University, vol. 22, no. 6, pp. 23-28, 2009. 
[8] W. Q. Liu, "An analysis of College Students' Cross-cultural Pragmatic Failures and its Strategies," Journal of Hubei Radio \& Television University, vol. 32, no. 9, pp. 134-135, 2012. 